\title{
Gestión de la emergencia ante eventos de inundación por tsunami en Chile: el caso de Puerto Saavedra ${ }^{1}$
}

\author{
Tatiana Montenegro-Romero ${ }^{2}$ y Fernando Peña-Cortés ${ }^{3}$
}

\begin{abstract}
RESUMEN
La gestión de la emergencia ante un evento de inundación por tsunami en Chile fue estudiada en el área urbana de Puerto Saavedra, Región de La Araucanía. Este procedimiento consistió en tres etapas: (1) evaluación y zonificación del riesgo en base a la metodología propuesta por las Naciones Unidas (ONU), (2) ejecución de talleres de participación ciudadana según la metodología AIDEP, y (3) confección de cartografía indicando zonas de seguridad y rutas de evacuación. Los resultados mostraron dos categorías de riesgo: máximo, asociada al área urbana, y sin riesgo, asociada al cordón montañoso. La comunidad no percibió como máximo el riesgo de inundación por tsunami, lo que dependería de la localización de las unidades vecinales y de la priorización de los riesgos más inmediatos. La aproximación de este estudio es de utilidad para la actualización de planes de protección civil en zonas litorales, experiencia que puede ser replicada en comunas costeras del país y Latinoamérica.
\end{abstract}

Palabras clave: Tsunami, riesgos naturales, plan protección civil, participación ciudadana, mapa de riesgo.

\begin{abstract}
Emergency management of tsunami floods in Chile was studied in the urban area of Puerto Saavedra, Araucanía Region. This procedure consisted of three stages: (1) risk assessment and zoning based on the methodology proposed by the United Nations (UN), (2) implementation of citizen participation workshops, according to the AIDEP methodology, and (3) implementation of maps indicating safety areas and evacuation routes. The results showed two risk categories: maximum, associated to the urban area, and no risk, associated to mountain range. The community did not perceive the risk of flooding due to tsunami as a maximum, which depended on the location of housing developments and the prioritization of the most immediate risks. The approach of this study is useful for updating civil protection plans in coastal areas, an experience that can be replicated in the coastal districts of the country and the rest of Latin America.
\end{abstract}

Key words: Tsunami, natural hazards, civil protection plan, citizen participation, risk map.

1 Artículo recibido el 1 de diciembre de 2009 y aceptado el 14 de septiembre de 2010.

2 Escuela de Ciencias Ambientales, Universidad Católica de Temuco (Chile). E-mail: tmontener@gmail.com
3 Laboratorio de Planificación Territorial, Universidad
Católica de Temuco (Chile). E-mail: fpena@uct.cl 
Los desastres naturales representan un problema ampliamente extendido a escala global, ya que suponen un alto riesgo para un mundo sostenible, tanto en su dimensión social como en la económica, es por esto que se han convertido en un problema central y crítico en las economías regionales (AyalaCarcedo et al., 2006; Zou \& Wei, 2009). En las últimas décadas, la vulnerabilidad a las amenazas naturales ha aumentado dramáticamente en América Latina y el Caribe, como consecuencia de una expansión urbana rápida y desordenada (BID, 2005), es así que la región conoce muy bien la devastación ocasionada por inundaciones, terremotos, huracanes, erupciones volcánicas, deslizamientos de tierra y sequías. En los últimos años, las amenazas naturales han dejado un saldo de más de 45.000 muertos, 40 millones de damnificados y daños que superan los US\$ 32.000 millones (Clarke y Pineda, 2007).

Los peligros naturales condicionan la capacidad de acogida del territorio, ya que al activarse pueden producir efectos indeseados en las actividades humanas (Gómez, 1994). Por ello, la prevención es fundamental para minimizar las pérdidas materiales y humanas, la que debe ser socializada e incluida en las políticas públicas (Wamsler, 2007). En este marco, el planteamiento básico de cualquier política de reducción de daños en un Estado moderno, al igual que sucede con los riesgos naturales, debe ser preventivo, por lo tanto, lo que debe minimizarse es el riesgo: la pérdida esperable (Ayala-Carcedo, 2001). Sin duda, las políticas actuales dan énfasis casi solamente a las actividades de respuesta a emergencias, las cuales gozan de una mayor visibilidad pública que la prevención. Esto ocurre porque los recursos son limitados y, en muchos casos, la ayuda posterior al desastre para la reconstrucción, proveniente de fuentes externas, ha actuado como un desincentivo para invertir en la reducción del riesgo (BID, 2005; Zhai \& Ikeda, 2006; CEPAL, 2007). En este sentido, es que en el Marco de la Acción de Hyogo, para los años 20052015, se solicita a los gobiernos considerar la evaluación de los riesgos de desastre en los planes de urbanismo y la gestión de los asentamientos humanos expuestos a desastres, donde deben tratarse prioritariamente los problemas de la vivienda precaria o provisional y la ubicación de las viviendas en las zonas de alto riesgo que prevean la inclusión de medidas estructurales y no estructurales para la gestión de desastres (EIRD, 2005; Wamsler, 2007).

El instrumento teórico para una mitigación racional y preventiva de los desastres naturales se denomina Evaluación de riesgo (Ayala-Carcedo et al., 2006), entendiendo riesgo como "el producto de la probabilidad de ocurrencia de un desastre, por la vulnerabilidad en tanto por uno y la exposición" (Ayala-Carcedo et al., 2006: 21), esto significa que al aumentar la probabilidad de ocurrencia, la vulnerabilidad y/o la exposición, implica que el riesgo aumenta en una relación directamente proporcional. Siguiendo este fundamento, Ayala-Carcedo (1993) considera tres factores de importancia que determinan el riesgo: la peligrosidad del medio físico, la vulnerabilidad del medio construido y la exposición de la población.

Según el World Institute for Disaster Risk Management (DRM) (BID, 2003), hay distintas maneras de manejar los riesgos frente a amenazas naturales, entre las que se encuentra la planificación territorial del uso de la tierra (por ejemplo exclusión de asentamientos humanos en zonas peligrosas), medidas preventivas, de emergencia y de recuperación o rehabilitación (basadas en una detallada planeación de la emergencia, la que debe continuar mientras transcurra la situación desastrosa), la transferencia de riesgos mediante seguros y el reconocimiento y aceptación del riesgo por parte de la comunidad (mediante el desarrollo de responsabilidades individuales). De este modo, un sistema nacional para el manejo de los desastres naturales, debe comprender una interacción entre medidas institucionales, mecanismos financieros y una población informada y con alternativas claras de acción frente a los mismos, teniendo en cuenta los conflictos de intereses y los diferenciados niveles de percepción frente al riesgo (CEPAL, 2007).

La gestión de la emergencia debe ser un proceso planificado, concertado, participativo e integral de reducción de las condiciones de riesgo de desastres (Chuquisengo y Gamarra, 2001), por ello es necesario establecer vías de comunicación entre los investigadores, las autoridades competentes, 
los técnicos dedicados a la determinación del riesgo y los vecinos afectados por los mismos, del tal modo que las propuestas de todos los sectores sean consensuadas e incorporadas en la planificación para un desarrollo local sostenible (Ferradas, 2004). En este contexto, la elaboración participativa de mapas de riesgos por parte de las autoridades, vecinos y encargados de la gestión y manejo de riesgo puede ser de gran ayuda para la toma de decisiones en cuestiones de reglamentación, reordenamiento urbano, zonificación y planes de emergencia (Arauz et al., 2007; Convertito et al., 2010). Es así como la percepción de la población en torno a los riesgos, el reconocimiento de su historia local, y su contribución en la identificación de zonas de amenazas, inciden decididamente en la organización, en las tecnologías aplicadas, el acceso a recursos y en la capacidad de gestión con y ante las autoridades e instituciones (Ferradas, 2004; Santiago y Sagástegui, 2008). Las observaciones locales de la población no solo ayudan a los gestores de emergencias a corroborar los datos científicos y las interpretaciones derivadas de los sistemas basados en tecnología, sino que, cuando ocurren acontecimientos peligrosos como los tsunamis dichas observaciones locales pueden Ilegar a ser la fuente primaria o única para detectar el peligro y accionar la alarma (Hall, 2006).

Por lo tanto, las actividades de capacitación en base a talleres participativos y seminarios constantes son imprescindibles para mantener una población informada y activa en la gestión de la emergencia, ya que el objetivo principal del plan es proteger $y$ salvar vidas. Bajo esta importante consideración, en el año 2002 el Gobierno de Chile aprueba definitivamente el Plan Nacional de Protección Civil del Ministerio del Interior, que incluye aspectos preventivos, de mitigación, de preparación y alertamiento temprano, derogando el Decreto Supremo $N^{\circ}$ 155 de 1977 (Plan Nacional de Emergencia). Así, se responde a los compromisos internacionales suscritos en la Década Internacional de Reducción de Desastres Naturales y a la modernización del propio Sistema Nacional de Protección Civil (ONEMI, 2002).

La historia sísmica de Chile muestra que en numerosas ocasiones las costas han experimentado solevantamientos o hundimientos detectados a simple vista. Varios terremotos fueron acompañados además por tsunami, cuyo origen está relacionado justamente con deformaciones bruscas del fondo marino (Lagos, 2000), por lo que terrazas marinas a lo largo de nuestras costas atestiguan estos movimientos (Ramírez, 1986). Cabe mencionar, uno de los desastres naturales más importantes de la historia ocurrido en Chile, el terremoto y tsunami de 1960, con una magnitud nunca antes registrada instrumentalmente (Mw 9.5). Trece de las 25 provincias de Chile fueron afectadas sin incluir los daños a nivel internacional en las ciudades y localidades costeras pertenecientes al océano Pacífico. Los efectos del desastre dejaron una profunda huella en la población y deterioraron seriamente la economía (OPS/OMS, 1994; Cisternas et al., 2005; Veyl, 1961; Sievers et al., 1963; Atwater, 1999; SHOA, 2000). Todos los pueblos costeros entre los $36^{\circ}$ y $44^{\circ}$ grados de latitud sur fueron destruidos o dañados fuertemente por la acción de las ondas del tsunami y el sismo (Lagos, 2000). Si bien este suceso ha sido el más recordado, en los últimos 30 años han ocurrido nueve importantes eventos sísmicos, los últimos en 2005 y 2007 en la zona norte del país, lo que reafirma la imperiosa necesidad de la prevención para minimizar las pérdidas materiales y de vidas humanas ante grandes desastres naturales (CEPAL, 2007).

Las costas son sistemas frágiles y esenciales en el desarrollo de la humanidad, por ello en áreas urbanas costeras expuestas al riesgo de inundación por tsunami se requiere una planificación adecuada de actividades y construcciones, estableciendo lugares de refugio y rutas de evacuación que permitan la prevención y mitigación este riesgo (Lavell, 2000; Lagos y Cisternas, 2008). Los proyectos de vivienda y asentamientos humanos debieran ser diseñados en beneficio de la prevención, habilitando zonas de escape en caso de emergencia, en un marco que permita el diseño de verdaderos asentamientos y no solo conglomerados de casas, considerando vías y sistemas de escape para la mitigación de probables impactos (Arguello, 2004). También es pertinente el establecimiento de áreas de refugio en los sectores pertenecientes a la plataforma o cordón montañoso en sectores aledaños a las ciudades, habilitando caminos hacia zonas de seguridad, como así también 
la instalación de señalética básica, donde la población reconozca las zonas de evacuación y seguridad.

En Chile, la gestión de la emergencia en caso de desastres se inicia con la activación de un Mando Conjunto a través del cual se adoptan las decisiones para que cada una de las autoridades cumpla con su rol, los diversos actores son divididos de acuerdo a la función estipulada en el plan de contingencia y respuesta, encontrando para tales casos un mando de autoridad (Intendencia, Gobernación, Servicio Hidrográfico y Oceanográfico de la Armada de Chile), un mando de Coordinación (ONEMI nacional y Regional, Oficina Comunal de Protección Civil, Municipalidad) y un mando Técnico (Bomberos, Carabineros, Seremis, Cruz Roja, Defensa Civil) (ONEMI, 2001). En este marco, se establece que el único organismo técnico encargado de la entrega de la información de un posible tsunami en las costas de Chile es el Servicio Hidrográfico y Oceanográfico de la Armada de Chile (SHOA), institución que tiene la obligación, después de confirmada la emergencia con el International Tsunami Information Center (ITIC), de informar a la Oficina Nacional de Emergencia del peligro real que posee este evento en las costas del país, el cual dará información a los diversos mandos de coordinación, para la activación de los planes de contingencia y alerta a la población (SHOA, 2000; SHOA, 2001).

La Región de La Araucanía, por su conformación y ubicación geográfica, está expuesta permanentemente a diversas situaciones de emergencia o catástrofe que pueden afectar a una o varias comunas (Intendencia Región de La Araucanía, 2004). Uno de estos fenómenos es el tsunami, que si bien se presenta con baja frecuencia posee una gran magnitud y efectos catastróficos en la población e infraestructura, como quedó en evidencia en el terremoto y tsunami de 1960, evento que provocó el hundimiento de 1,2 metros, dejando a la comuna de Puerto Saavedra prácticamente bajo el agua (SHOA, 2000). En este marco, y entendiendo la importancia de la prevención del riesgo de inundación por tsunami en Chile, se plantea que la gestión de la emergencia ante un evento de inundación por tsunami puede abordarse participativamente mediante la evaluación de la peligrosidad natural, la vulnerabilidad y la exposición. Para probar esta hipótesis, en este estudio se propone el objetivo de evaluar la gestión de la emergencia y la percepción de la población frente al riesgo en el área urbana de Puerto Saavedra, antecedentes que pueden ser utilizados en la actualización e implementación de planes de prevención a corto y mediano plazo.

\section{Materiales y métodos}

\section{Área de estudio}

El área de estudio corresponde al asentamiento urbano de Puerto Saavedra, se ubica entre los $38^{\circ} 47^{\prime} 16^{\prime \prime}$ de Latitud Sur, y entre los $73^{\circ} 23^{\prime} 47^{\prime \prime}$ de Longitud Oeste en el borde costero de la Región de La Araucanía (Figura $\mathrm{N}^{\circ} 1$ ). Se incluyen, además, las zonas de seguridad en el área periférica. La población total de la comuna es de 14.034 habitantes de los cuales 2.679 pertenecen al área urbana, siendo su superficie censal $1,7 \mathrm{~km}^{2}$, con un total de 814 viviendas (INE, 2003).

\section{Gestión de la emergencia ante inundación por tsunami}

El procedimiento de trabajo se dividió en tres etapas: la evaluación del riesgo de inundación, la percepción del riesgo mediante participación ciudadana y la zonificación cartográfica del riesgo. La evaluación del riesgo de inundación por tsunami se efectuó de acuerdo a la metodología propuesta por la Organización de las Naciones Unidas (Ayala-Carcedo, 1993) adaptada por AyalaCarcedo y Olcina (2006). Originalmente el procedimiento consiste en tres etapas, la primera denominada inventario y evaluación de riesgos (identificación del riesgo), la segunda denominada análisis de riesgos (aceptabilidad social y económica), y la tercera consiste en el análisis de la reducción del riesgo (medidas de mitigación). En este estudio solo se consideró la primera etapa de esta metodología, puesto que fue necesaria la construcción de la carta de riesgo de inundación por tsunami, como herramienta base para la gestión de la emergencia, la que evaluó los factores de riesgo del territorio y que fueron socializados a través de talleres de participación ciudadana. El método para la contrucción de la carta de riesgo integró tres factores: exposición, 
vulnerabilidad y peligrosidad natural. Para Puerto Saavedra, se consideró la densidad de población como única variable dentro del factor de exposición, el tipo de materiales de construcción y número de viviendas dentro del factor de vulnerabilidad, y la geomorfología, pendiente y altitud, dentro del factor de peligrosidad natural. Finalmente, la integración de los factores y variables fueron agregadas cartográficamente utilizando el programa ArcGis 9.2, que originó la carta de inundación por tsunami.

Esta metodología fue aplicada en el marco del Proyecto Fondecyt No 1030861: "Análisis integrado del Borde Costero de la Región de La Araucanía. Propuestas y criterios para la planificación ecológica de sus humedales", donde se categorizó el riesgo en cuatro clases: máximo, medio, mínimo y nulo (PeñaCortés et al., 2004; Montenegro-Romero, 2006). En el caso de Puerto Saavedra, sin embargo, debido a la reducida área de estudio, donde el asentamiento urbano concentra la mayor parte de las actividades económicas y sociales, se utilizó el criterio de presencia o ausencia de población e infraestructura. Esto determinó que los factores de exposición y vulnerabilidad se clasifiquen únicamente en dos categorías: sin riesgo y máximo riesgo.

Figura $\mathrm{N}^{0} 1$

Ubicación área de estudio

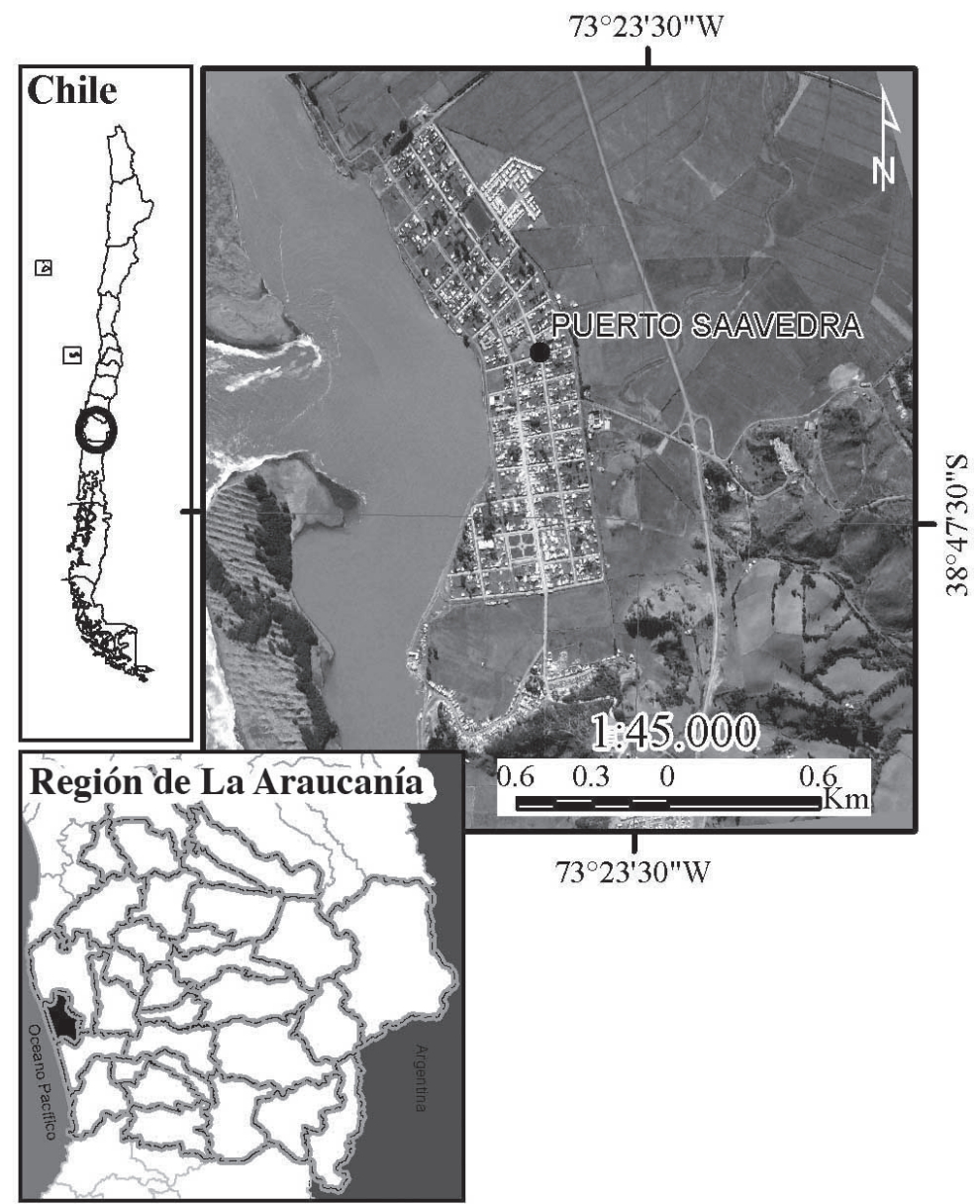

Fuente: Elaboración propia. 
Similar situación ocurre con el factor de peligrosidad natural, el que considera la geomorfología como uno de los elementos más relevantes para la categorización del riesgo, en este sentido en el área de estudio solo se observan tres unidades principales, la Ilanura aluvial, las terrazas y el cordón montañoso (Peña-Cortés et al., 2006), lo que determina su clasificación en las categorías sin riesgo y máximo riesgo, respectivamente. Adicionalmente, considerando el peor escenario, la altura máxima del tsunami en 1960 no habría superado los 10 m en Puerto Saavedra (Monge, 1993) y, en general, la cota máxima de inundación para las costas del centro sur de Chile no superaría los 20 a 25 m.s.n.m. (Lagos, 2000; Lagos y Cisternas, 2008).

La segunda etapa se basó en la aplicación del método AIDEP (análisis histórico, investigación en terreno, discusión y análisis, elaboración del mapa, plan), planteado por la Oficina Nacional de Emergencia (ONE$\mathrm{MI})$, técnica nacionalmente conocida y utilizada por las oficinas de protección civil a nivel municipal, para el desarrollo de planes de preemergencia de forma participativa (ONEMI, 2001).

La participación local en la detección de los riesgos se basó en un conjunto de actividades que incluyeron tres reuniones-taller con dirigentes sociales pertenecientes a las cuatro juntas vecinales del área urbana y dos reuniones con la persona encargada de la Unidad de Protección Civil y Cruz Roja, además del trabajo de campo con los propios vecinos y dirigentes. El propósito de esta actividad fue recoger las percepciones acerca del riesgo y compartir criterios técnicos y sociales para su identificación, elemento que permitió la realización de un listado de los riesgos presentes en el territorio, el que posteriormente fue priorizado por las propias juntas vecinales. Desde el punto de vista práctico, la metodología contempló el trabajo con dos grupos o tipos de actores, los vecinos, representados a través de sus juntas de vecinos, y el municipio, representado a través de la unidad de Protección Civil y Cruz Roja. De esta manera, se entiende la participación ciudadana como un proceso de intercambio de información entre agentes técnicos y actores locales, cuyo propósito es la construcción de un conocimiento y/o levantamiento de iniciativas orientadas al impacto local. El enfoque teórico-metodológico de esta etapa del estudio se encuentra en la investigación social participativa (Canales, 2006) y en la propia metodología AIDEP.

La tercera etapa consistió en la construcción de cartografía a escala 1:5.000, donde fue posible visualizar en detalle las zonas de seguridad y rutas de evacuación. Para ello se realizó un levantamiento en terreno con el apoyo de Cartografía IGM de Puerto Saavedra escala 1:50.000 y fotografía aérea escala 1:20.000 vuelo SAF Fondecyt No 1030861 (2004), la información generada fue digitalizada y editada en el sofware ArcGis 9.2. En esta fase se realizó un taller final de participación donde se presentaron los resultados del proceso de investigación, en especial los referidos a las alternativas de evacuación frente a un tsunami. Finalmente se presenta una secuencia de fotografías áreas de los años 1960, 1992 y 2009 donde es posible visualizar la expansión urbana y las modificaciones del territorio.

\section{Resultados y discusión}

\section{Nivel de riesgo y percepción ciudadana}

La evaluación de los factores de riesgo determinó que la exposición y vulnerabilidad en el área urbana fueran máximos, justificado por la alta densidad poblacional (73,71 hab/ $\mathrm{km}^{2}$ ), con una densidad de viviendas de 10 casas $/ \mathrm{km}^{2}$, además, se advierte la presencia de establecimientos educacionales, servicios básicos, infraestructura vial, obras portuarias y servicios administrativos emplazados en áreas de máximo riesgo. Asimismo, se constató que la mayor vulnerabilidad se encuentra asociada a zonas con construcciones que no cuentan con fijaciones en sus estructuras y que se distribuyen en posición paralela a la línea de la costa; aquí cabe mencionar que el potencial destructivo de un tsunami es el resultado de la inundación debido al impacto de las olas sobre las construcciones y posterior erosión (Lagos et al., 2008). Así, la superficie de riesgo máximo de inundación por tsunami en el área urbana de Puerto Saavedra Ilegó a 330,8 ha, equivalente al 69\% de área total evaluada. Esto se explica por la alta peligrosidad natural del territorio, el que se encuentra geomorfológicamente ubicado sobre 
terrazas y llanuras fluviomarinas (Figura $\mathrm{N}^{\circ}$ 2B). Además, se observó la presencia de humedales ribereños formados por el terremoto de mayo de 1960, el que entre otros efectos generó el hundimiento del terreno.

Esta situación se agrava si se considera la cercanía de la desembocadura del río Imperial y cursos de aguas superficiales que rodean el área urbana. Por otra parte, el área sin riesgo, que corresponde a 148,2 ha $(31 \%)$, se justifica por la presencia de un cordón montañoso perteneciente a la cordillera de la Costa y a la baja densidad poblacional e infraestructura en esta área, lo que condiciona el lugar como un área de seguridad y refugio potencial (Figura $\mathrm{N}^{\circ} 2$ ).

Desde el punto de vista de la evaluación del medio físico, Mardones y Vidal (2001) establecen que los sectores más amenazados frente al riesgo de inundación por tsunami son las Ilanuras aluviales y las terrazas inferiores hasta aproximadamente $2 \mathrm{~km}$ de la desembocadura de los ríos, por lo tanto, el impacto de un tsunami variará sensiblemente de acuerdo a la topografía costera, debido a que cuando la pendiente es relativamente fuerte la extensión de la zona inundada no es significativa, al contrario de lo que ocurre cuando el terreno es plano o con escasa pendiente, donde la inundación resultante se puede extender tierra adentro por más de 300 $\mathrm{m}$ cubriendo grandes extensiones de agua y escombros (Lagos, 2000; ONEMI, 2001).

El recuerdo histórico de lo vivido en el tsunami de 1960, sensibiliza y crea conciencia en la población de Puerto Saavedr, frente al riesgo al que se exponen, lo que queda

Figura $N^{\circ} 2$

Zonificación del riesgo de inundación por tsunami (A) y unidades geomorfológicas en Puerto Saavedra (B)

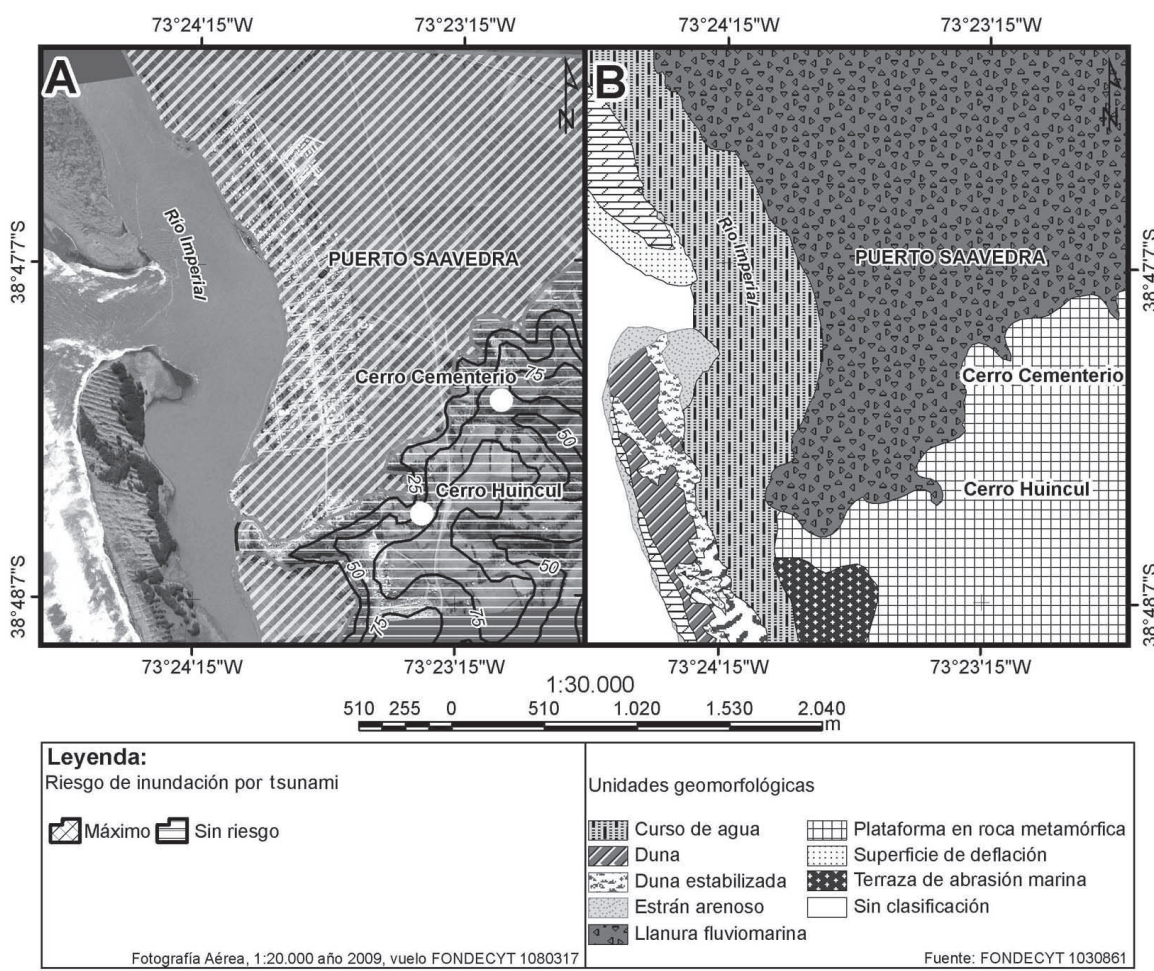

Fuente: Elaboración propia. 
demostrado en el trabajo de participación ciudadana realizado con las juntas vecinales (Cuadro $N^{\circ} 1$ y Figura $N^{\circ} 3$ ) donde se prioriza dentro de sus problemas inmediatos el riesgo de inundación por tsunami. Sin embargo, este riesgo natural no es considerado como de máxima importancia, puesto que aparecen otros más inmediatos y que los afecta en forma frecuente (por ejemplo inundación de calles y desbordes de ríos). Cabe mencionar que en la junta de vecinos La Ribera, la percepción del riesgo por tsunami cobró mayor importancia debido a que los participantes corresponden en su mayoria a pescadores que sobrevivieron al tsunami de 1960 (Figura $N^{\circ} 3$ ). Estos resultados se relacionan con la experiencia directa de la población frente a un desastre natural, ya que aquellas personas que nunca han estado expuestas a estos eventos naturales pueden percibir menor riesgo, y opuestamente, las experiencias emocionales fuertes motivan un comportamiento hacia la prevención (Siegrist \& Heins, 2006). Este comportamiento puede justificarse en la subestimación de los riesgos locales ya que pueden ser catalogados como fenómenos extremos o raros (Burningham et al., 2008). De este modo, el grado de sensibilización de la población además del tipo concreto de problemas cotidianos que inquietan a la opinión pública, influyen en el tipo de respuesta (García y Silió, 2001; Siegrist \& Heins, 2006), otros aspectos como la forma de vida de la población, el grado de exposición del área geográfica, las características de sus viviendas y su organización familiar (Arauz et al., 2007) son consideraciones relevantes como base para establecer el grado de percepción de la población frente al riesgo al que se encuentran expuestos.

Las juntas vecinales y organizaciones sociales son un espacio importante de participación ciudadana, debido a que se relacionan personas diversas con un interés común para la realización de una acción determinada, posibilitando el intercambio de experiencias que facilitan los procesos para una acción consensuada (Aedo et al., 2010; Mitchell et al., 2010). La percepción del riesgo y la "cultura de riesgo" son importantes factores determinantes de la existencia y del éxito de las políticas públicas, ya que permiten la sensibilización y el fomento de la capacidad de comprensión de los desastres naturales (Nathan, 2008).

\section{Cuadro $\mathrm{N}^{\circ} 1$}

Priorización en orden descendente del riesgo de inundación por tsunami

\begin{tabular}{|l|l|}
\hline \multicolumn{1}{|c|}{ Junta vecinal } & \multicolumn{1}{|c|}{ Prioridad del riesgo } \\
\hline Palmeras $N^{\circ} 4$ & $\begin{array}{l}\text { Desborde de río } \\
\text { Inundación de calles } \\
\text { Tsunami }\end{array}$ \\
$N^{\circ} 1$ Cornelio Sepúlveda & $\begin{array}{l}\text { Desborde de río e inundación por las vegas } \\
\text { Tsunami }\end{array}$ \\
& Desborde del canal \\
No 2 Agusto Winter & Inundación por marejadas altas \\
& Derrumbe de sitios \\
& Tsunami \\
$N^{0} 2$ Sector La Ribera & Tsunami \\
& Inundación \\
& Anegamiento \\
\hline
\end{tabular}

Fuente: Elaboración propia. 
Figura $\mathrm{N}^{\circ} 3$

Localización de las juntas vecinales de Puerto Saavedra

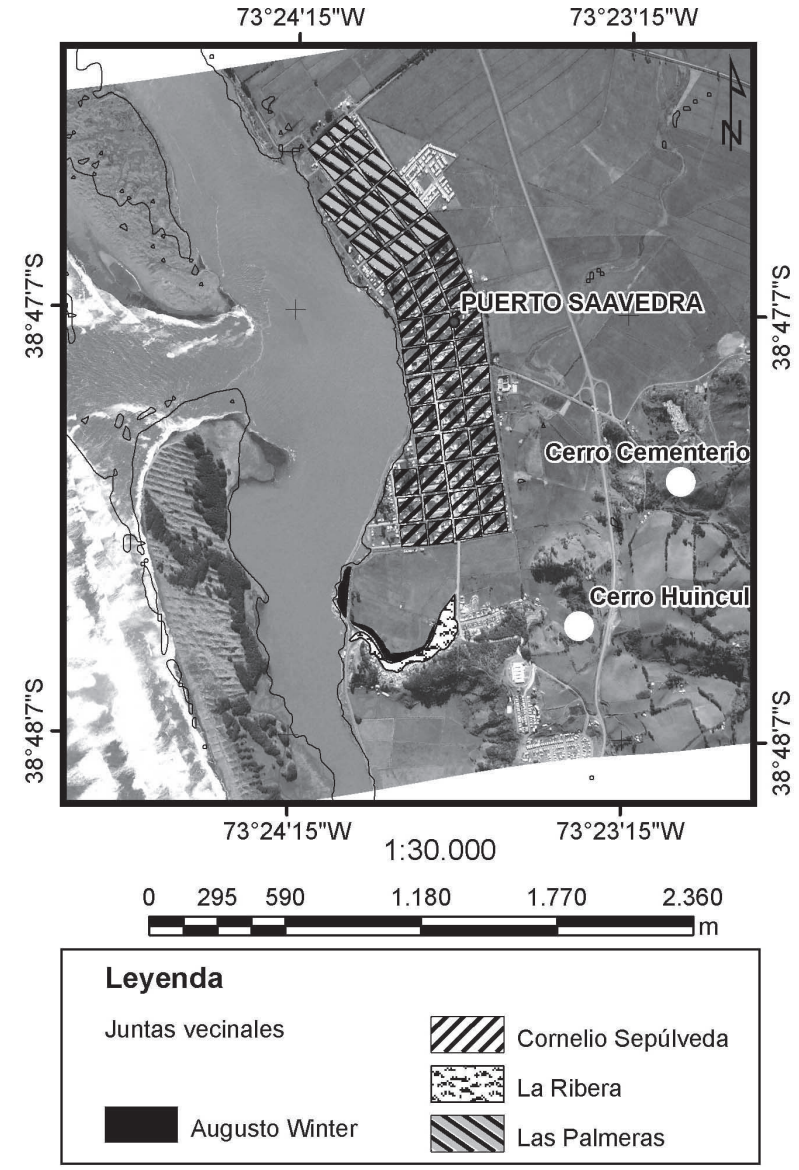

Fuente: Elaboración propia.

En este marco, cabe destacar el último simulacro de tsunami realizado por la OREMI Región de La Araucanía y el encargado de protección civil comunal en Puerto Saavedra en conjunto con las autoridades locales, regionales y Fuerzas Armadas en octubre del año 1999. Esta actividad tuvo por finalidad evaluar el grado de percepción de la población frente al plan, para crear conciencia del daño al cual se encuentran expuestos y reconocer los sitios de evacuación pertinentes. La posterior evaluación del plan detectó falencias operativas del mismo, entre las que se encuentran problemas en las comunicaciones, coordinación de puestos de mando y falta de mantenimiento en las vías de evacua- ción. Entre los aspectos positivos se enuncia el alto porcentaje de participación por parte de la comunidad (64\%) y de las instituciones comunales, que lograron realizar las funciones y acciones previamente establecidas en el plan, finalizando la evacuación total del pueblo en un tiempo estimado de 27 minutos (OREMI, 1999). Sin embargo, dicha actividad requiere ser desarrollada con mayor frecuencia.

En Chile, la gestión de la emergencia en caso de desastre se inicia con la activación de un Mando Conjunto a través del cual se adoptan las decisiones para que cada una de las autoridades cumpla con su rol. Es en esta 
dinámica donde se manifiesta confusión por parte de la ciudadanía, si bien se percibe un reconocimiento de actores clave, un $45 \%$ de las personas participantes no poseen claridad de cómo o qué instancia institucional entrega la información. Ejemplo de esto es lo ocurrido en el falso tsunami de Concepción el año 2005 y el falso tsunami de Tongoy en el año 2009 (Diario El Sur, 2006; Diario La Nación, 2005; Diario el Día, 2009), donde mediante un aviso informal se alertó a la población en forma innecesaria, provocando pánico y desconcierto total. Por otro lado, queda de manifiesto la tardía reacción y descoordinación ante el terremoto del 27 de febrero de 2010 (Diario El Mostrador, 2010).

\section{Áreas de seguridad y de evacuación}

El trabajo cartográfico y de participación ciudadana permitió definir rutas de evacuación identificando calles y vías de acceso a zonas de seguridad (Cuadro $N^{\circ} 2$ y Figura $N^{\circ}$ 4). Se definieron dos zonas de seguridad: el cerro Cementerio (zona I), considerado como el centro general de operaciones, y el cerro Huincul (zona II), los que forman parte de los cordones litorales ubicados sobre los 40 metros de altura. Los mismos sirvieron como áreas de seguridad en el terremoto y tsunami de mayo 1960. Las zonas de seguridad son lugares que se encuentran fuera del área de riesgo de inundación y deben ser espacios físicos amplios, que permitan recibir a toda la población evacuada (ONEMI, 2001). En el área de estudio las zonas de seguridad y resguardo poseen las condiciones de accesibilidad adecuadas, tanto para el acceso de personas como para el establecimiento de albergues temporales y servicios básicos de asistencia incluidos dentro del plan de contingencia frente al riego de inundación por tsunami.

Las vías de evacuación definidas corresponden a calles, rutas o caminos comunales y privados designados para el desplazamiento de la gente evacuada hacia las zonas de seguridad. Mientras dure la emergencia, por estas vías no deben circular por ningún motivo vehículos. En tiempos de normalidad es recomendable que estas calles tengan flujo permanente en dirección a las zonas de seguridad y no hacia la línea de costa (ONEMI, 2001), es por esta razón que se sugiere implementar medidas estructurales, como proyectos de mantención y pavimentación de caminos y ensanchamiento de puentes de acceso a las zonas de seguridad.

\section{El crecimiento urbano y las} modificaciones en el territorio

A partir de la destrucción completa de Puerto Saavedra luego del terremoto y tsunami de 1960 (Lagos, 2000) (Figura $N^{\circ} 5 \mathrm{~A}$ ), el crecimiento urbano hasta el año 2000 fue enfocado hacia al establecimiento de nuevas poblaciones en el cerro Huincul, donde además se inauguró a mediados del año 2007 el Hospital Intercultural Arturo Hillerns (Figura $N^{\circ}$ 5C) ubicado en la subida de la calle Los Araucanos, reemplazando su antigua ubicación en calle 18 de Septiembre (Figura $N^{\circ} 5 \mathrm{~B}$ ), la que estaba inserta en un área de máximo riesgo. Más recientemente, la inversión pública en viviendas sociales a partir del año 2007 en el área urbana de Puerto Saavedra se ha materializado a escasos metros de la línea de la costa y a solo 3 m.s.n.m. (por

Cuadro $\mathrm{N}^{\circ} 2$

Principales vías de acceso a zonas de seguridad

\begin{tabular}{|c|c|c|c|}
\hline Zona de seguridad & $\begin{array}{c}\text { Altitud } \\
\text { (m.s.n.m.) }\end{array}$ & $\begin{array}{c}\text { Coordenadas } \\
\text { geográficas (UTM) }\end{array}$ & Vías de acceso \\
\hline Cerro Cementerio (zona I) & 42 & $0640.270-5.705 .280$ & $\begin{array}{l}\text { Calle San Sebastián } \\
\text { por calle } 21 \text { de Mayo }\end{array}$ \\
\hline Cerro Huincul (zona II) & 79 & $0639.709-5.704 .189$ & $\begin{array}{l}\text { Subida calle Los Araucanos } \\
\text { por calle Esmeralda }\end{array}$ \\
\hline
\end{tabular}

Fuente: Elaboración propia. 
ejemplo villas La Costa y Los Cisnes) (Figura $\mathrm{N}^{\circ} 5 \mathrm{C}$ ), sin considerar el alto riesgo al cual se encuentra expuesta la población. Esto es posible debido a un plan regulador comunal permisible y obsoleto que data del año 1964. La escasa consideración de la dinámica y recurrencia de procesos naturales extremos y su relación con el emplazamiento de viviendas puede tener consecuencias no deseadas, induciendo el riesgo de desastre (Lagos y Cisternas, 2008). Un aspecto a considerar son los avances en la conectividad de la ciudad, donde es posible visualizar la carretera de la costa (Ruta S 282) (Figura $N^{\circ} 4$ ), pavimentación de caminos (por ejemplo calle que conecta al nuevo hospital), la instalación de una costanera y la construcción de infraestructura turística en la caleta de pescadores.

Figura $\mathrm{N}^{\circ} 4$

Calles y vías de acceso a zonas de seguridad

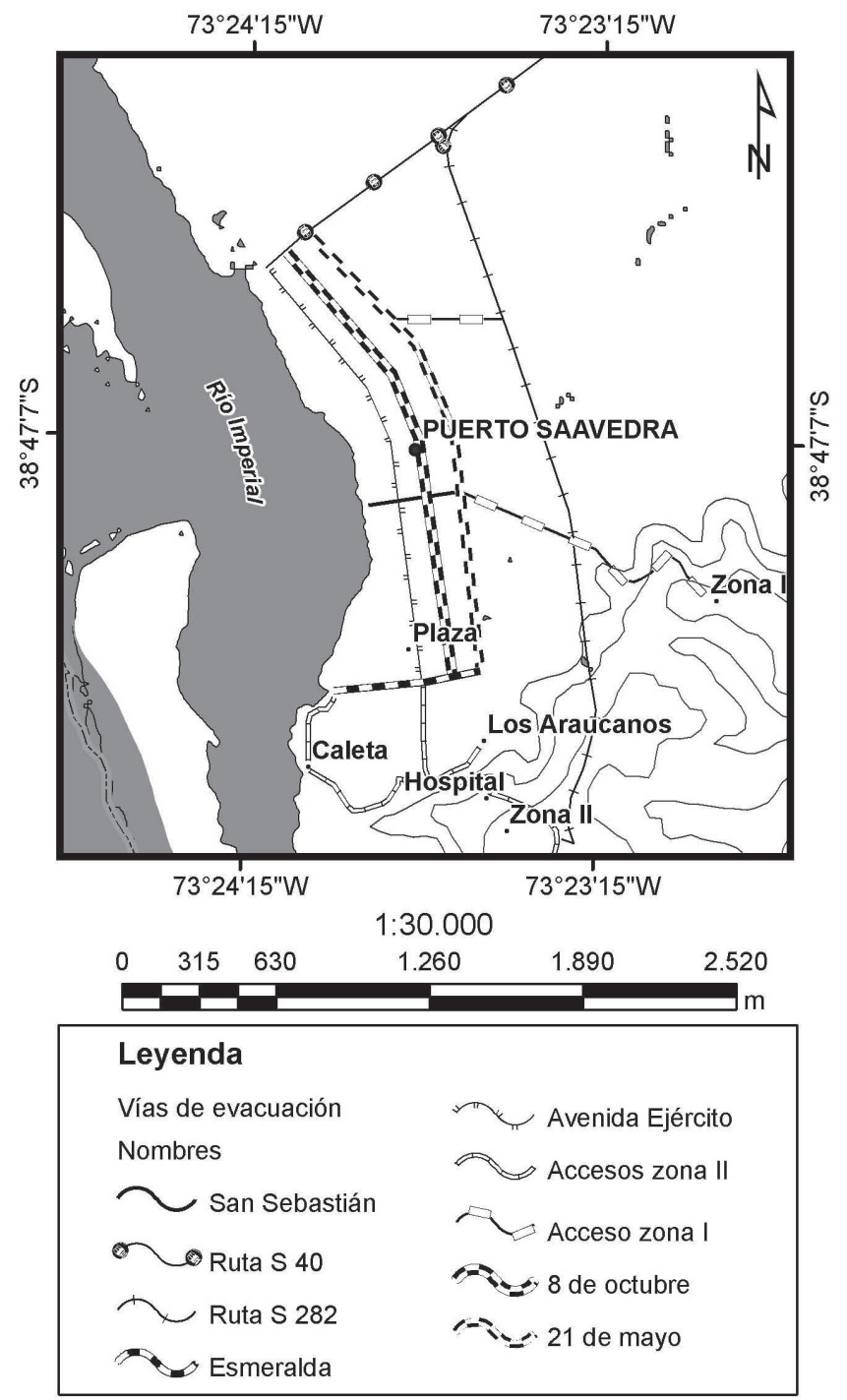

Fuente: Elaboración propia. 
Es importante considerar que en mayo de 2009, en la ordenanza de la Ley General de Urbanismo y Construcciones (Artículo 2.1.17) se estableció que en los planes reguladores podrán definirse áreas restringidas al desarroIlo urbano, por constituir un peligro potencial para los asentamientos humanos. En estas áreas, el plan regulador establecerá las normas urbanísticas aplicables a los proyectos una vez que cumplan con los requisitos establecidos en este inciso. Lo anterior es un gran avance a nivel nacional en el área de protección civil, puesto que al aceptar la realidad del territorio y su diversidad geográfica permite mejorar la gestión del riesgo de desastre.

Desde la perspectiva territorial se verifica el hundimiento del terreno en más de 1,2 m luego del gran sismo (SHOA, 2000). Además, se observan modificaciones tanto en el cauce del río Imperial como en la configuración espacial de la barra y la erosión general de la línea costera (Figura $N^{\circ}$ 5), la cual se ha controlado en parte con plantaciones de Pinus radiata (Peña-Cortés et al., 2008). Cabe mencionar que Puerto Saavedra sigue en el mismo sitio que fue destruido inicialmente, a diferencia de lo ocurrido con el asentamiento urbano de Toltén, el cual fue reconstruido $2 \mathrm{~km}$ hacia el interior luego del terremoto de 1960.
El ordenamiento territorial de asentamientos costeros y los modelos de inundación por tsunami son un aspecto integral para la planificación y estudios de riesgo. La delimitación de áreas de inundación se debería traducir en información crítica al momento de definir potenciales usos de suelo, vías de evacuación y localización de zonas de seguridad (Lagos y Gutiérrez, 2005). De acuerdo a lo anterior, las cartas de inundación por tsunami confeccionadas mediante modelos de simulación numérica por el Servicio Hidrológico y Oceanográfico de la Armada de Chile (SHOA) sin duda son un gran avance para planificación territorial, sin embargo, la obtención de estos mapas a nivel comunal y regional es de alta dificultad para ciudades como Puerto Saavedra, debido a la falta de datos básicos necesarios para la aplicación de este tipo de instrumentos (datos batimétricos, levantamientos topográfico de curvas de nivel y aerofotogrametría). Además, esta situación posiblemente responda a que es un área que decrece económicamente y registra altos índices de pobreza y emigración de población (Peña-Cortés et al., 2009). Adicionalmente, no posee un puerto comercial de importancia económica y productiva, a diferencia de otras ciudades costeras de la zona centro-norte que presentan mayor desarrollo.

Figura $\mathrm{N}^{0} 5$

Modificaciones físicas y urbanas de Puerto Saavedra en los años 1960(A), 1992(B) y 2009 (C). La línea de la costa aparece en forma discontinua
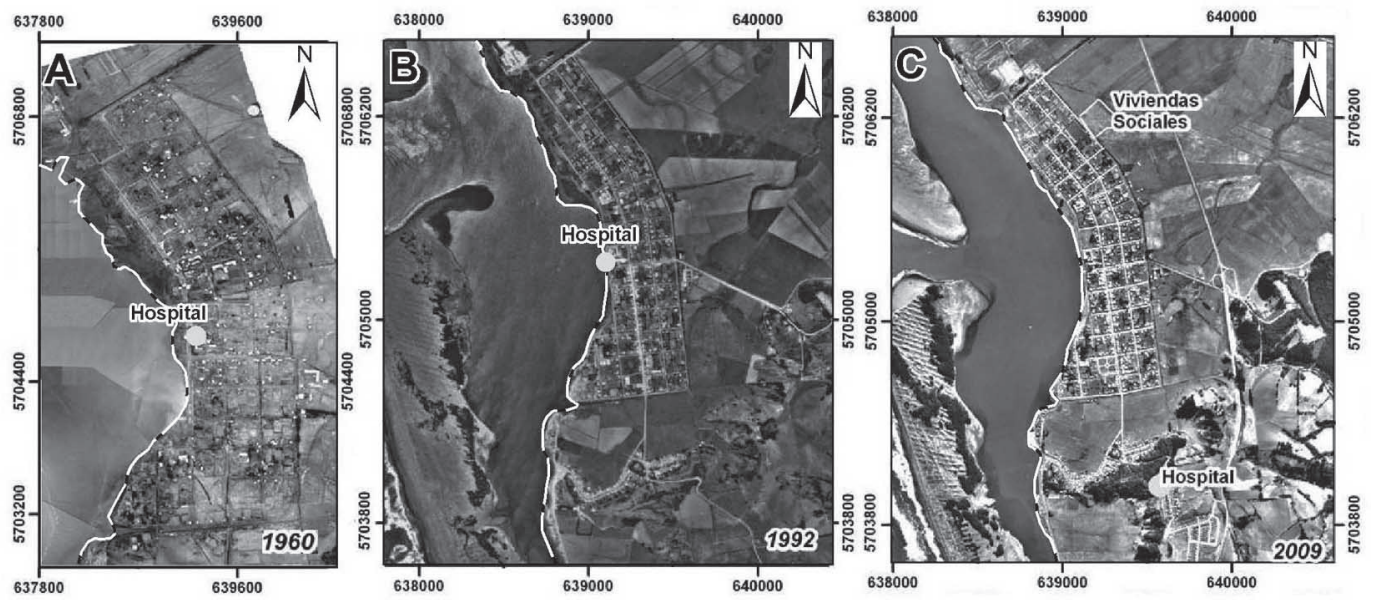

Fuente: Elaboración propia. 


\section{Consideraciones finales}

Considerando que los recursos económicos disponibles para la prevención de desastres son limitados, muchas de las comunas costeras de Chile se encuentran expuestas a una normativa permisible, a la urbanización de sitios de alto riesgo (menor costo) y a un desconocimiento de su propia historia de eventos catastróficos. Efectivamente, en Chile aún son pocas las comunas costeras que han implementado zonas de restricción y protección en sus instrumentos de planificación urbana, en condiciones de fragilidad ambiental o en presencia de riesgos naturales. En estas áreas se ha restringido la intervención antrópica, como es el caso de las ciudades de Viña del Mar, Coquimbo y Antofagasta, comunas en las cuales se definen en sus ordenanzas municipales, zonas más propensas a los efectos de maremotos o tsunami (Municipalidad de Coquimbo, 2005; Municipalidad de Viña del Mar, 2005; Municipalidad de Antofagasta, 2008). Sin embargo, la zonificación no tiene representación areal en los planos de zonificación, y se orienta a la definición de restricciones y formulación de medidas de contingencia asociadas a niveles altimétricos de potencial inundación. Uno de los aspectos más importantes que han desarrollado dichos municipios es la inversión en programas de señalética vial que informa a los habitantes (permanentes y turistas) las vías de evacuación, zonas de seguridad y zonas de inundabilidad en la ciudad (Municipalidad de Coquimbo, 2005; Municipalidad de Viña del Mar, 2005; Municipalidad de Antofagasta, 2008), por tanto, los instrumentos de planificación pueden y deben tomar en cuenta los factores de riesgo al momento de regular el uso de los espacios. En efecto, es importante considerar las incidencias previsibles de la planificación sobre el medio ambiente $y$, particularmente, la prevención de los riesgos naturales (Nathan, 2008).

Por lo tanto, la planificación que otorgue especial importancia a la preparación y difusión de las acciones de prevención, permitirá aminorar o mitigar los efectos y consecuencias de una emergencia o catástrofe (Intendencia de la Región de La Araucanía, 2004). En este contexto es importante que las cartas de inundación por tsunami, que hasta la fecha han sido realizadas para 25 ciudades del país por el Servicio Hidrográfico y Oceanográfico de la Armada (proyecto Cartas de Inundación por Tsunami), sean incluidas en el proceso de zonificación de los planes reguladores de ciudades costeras, donde se promueva la disminución de las densidades de habitantes en las zonas amenazadas, incentivando la localización de áreas verdes, evitando así la construcción de infraestructura crítica, como colegios, hospitales, cárceles y servicios en general (SHOA, 2000; SHOA, 2001; SHOA, 2002).

La evaluación de riesgos, el ordenamiento territorial, la planificación de asentamientos urbanos y la capacidad para tomar decisiones sobre nuestro entorno inmediato en un contexto de gobernabilidad son necesarios para el proceso de prevención y activación de un plan de contingencia. La clave de la reducción del riesgo está en el entendimiento del riesgo mismo, en la educación acerca de él y en la participación decidida y comprometida de todos los actores sociales, privados y públicos en su resolución (Edeso, 2008; Mitchel et al., 2010). Para ello, es fundamental la realización de actividades de capacitación constante, actualización de cartografías de riesgos y planes de contingencia. Así, en un plan de emergencia debiera disponerse un procedimiento de evaluación inmediata de la situación, así como de su evolución; para esto, un análisis previo de la vulnerabilidad en las zonas de alto riesgo facilitará la evaluación de los recursos necesarios para hacer frente a los efectos adversos de la emergencia, como servicios de rescate y salvamento, áreas de resguardo y alojamiento, preparación de hospitales, entre otros (Vidal et al., 2001).

Dado lo anterior, es clara la necesidad de establecer un sistema activo que permita entregar, entre otros elementos, el aviso oportuno sobre la probable ocurrencia o puesta en marcha de una situación o un hecho determinado. En este marco, la definición clara de los roles específicos que cumple cada actor inserto en un plan, debe ser fácilmente identificable por la comunidad y entre organismos públicos y privados, así como establecer claramente los roles y funciones que desarrollan en la implementación de un plan de emergencia. 
En conclusión, debe destacarse que el riesgo de inundación por tsunami en el asentamiento urbano de Puerto Saavedra es máximo $(69 \%)$ debido principalmente a las características geomorfológicas del territorio, alta densidad poblacional e infraestructura expuesta al riesgo, vulnerabilidad de las construcciones y la presencia de la mayoría de los servicios en áreas de alto riesgo.

El riesgo de inundación por tsunami es identificado por la comunidad de Puerto Saavedra (juntas vecinales), pero la evaluación de su impacto sugiere que depende de la localización de la población y de la priorización de los riesgos más inmediatos.

Las zonas de seguridad, como el cerro Cementerio y el cerro Huincul son sectores percibidos por la comunidad como zonas de refugio de acuerdo a experiencias anteriores $y$, por lo tanto, es necesario mantener estas vías de evacuación en buen estado y con indicaciones claras a través de señalizaciones previamente establecidas.

Finalmente, de acuerdo a la experiencia nacional e internacional en materia de riesgos naturales, se establece que el análisis del ciclo del riesgo debe ser participativo y organizado, estableciendo para ello recursos para la prevención, es decir, la población debe ser capacitada y educada periódicamente sobre el riesgo al cual están expuestos (por ejemplo talleres, charlas, seminarios y desarrollo de simulacros de evacuación).

\section{Agradecimientos}

A Jimmy Pincheira por la revisión crítica del manuscrito, a Osvaldo Almendra y Eduardo Fernández por la edición de cartografía y a Guido Cancino, encargado de la Oficina de Protección Civil de la comuna de Puerto Saavedra, por su valiosa contribución para el desarrollo de este trabajo. La presente investigación contó con el financiamiento de la Dirección General de Investigación y Postgrado de la Universidad Católica de Temuco, proyecto DGIUCT No 2007-DGI-TP-04 y del Proyecto FONDECYT No 1080317.

\section{Referencias bibliográficas}

AEDO, I.; DÍAZ, P.; CARROLL, J.; CONVERTINO, G. \& ROSSON M. Enduser oriented strategies to facilitate multiorganizational adoption of emergency management information systems. Information Processing and Management, 2010, vol. 46, p. 11-21.

ARAUZ, J.; MONTERO, C. y LAZO, R. Informe final Proyecto "Riesgos Naturales y Percepción". Campaña educativa en prevención de riesgos a desastres en cinco escuelas de Nicoya, Guanacaste, Costa Rica "Educar es Prevenir". Observatorio Vulcanológico y Sismológico de Costa Rica. Nicoya: Universidad Nacional OVSICORIUNA, 2007. Disponible en Internet: http:// www.ovsicori.una.ac.cr/Riesgos/documentos/ InfofinalproyectPreRiesgos-CONARE070307. pdf

ARGÜELLO-RODRÍGUEZ, M. Riesgo, Vivienda y Arquitectura. En: San Juan, Congreso ARQUISUR, 2004. Disponible en Internet: http://cidbimena.desastres.hn/docum/ crid/Febrero2006/CD-2/pdf/spa/doc15586/ doc15586-contenido.pdf

ATWATER, B.; CISTERNAS, M.; BOURGEOIS, J.; DUDLEY, W.; HENDLEY, J. \& STAUFFER, P. Surviving a tsunami: Lessons from Chile, Hawaii, and Japan. U. S. Geological Survey, circular No 1187, 1999. Disponible en Internet: http://pubs.usgs.gov/ circ/c1187/

AYALA-CARCEDO, F. Estrategias para la reducción de desastres naturales. Investigación y Ciencia, 1993, No 200, p. 6-13.

AYALA-CARCEDO, F. La ordenación del territorio en la prevención de catástrofes naturales y tecnológicas. Bases para un procedimiento técnico-administrativo de evaluación de riesgos para la población. Boletín de la A.G.E., 2001, No 30, p. 37-49.

AYALA-CARCEDO, F.; OLCINA, J.; LAIN, L. y GONZALES, A. Riesgos naturales y desarrollo sostenible: impacto, predicción y mitigación. Madrid: Instituto geológico y minero de España, 2006. 
AYALA-CARCEDO, F y OLCINA, J. (coords.). Riegos Naturales. Barcelona: Editorial Ariel Col. Ciencias, 2006.

BANCO INTERAMERICANO DE DESARROLLO (BID). Evaluation of InterAmerican Development Bank's Operational Policy on Natural and Unexpected Disasters. Canobbio: Virginia Tech, Board of the Swiss Federal Institutes of Technology, 2003. Disponible en Internet: http://www. drmonline.net/drmlibrary/pdfs/DRM02.pdf

BANCO INTERAMERICANO DEL DESARROLLO (BID). Gestión de riesgo de amenazas naturales en proyectos de desarrollo. Lista de preguntas de verificación "Checklist". Serie de informes de buenas prácticas del Departamento de Desarrollo Sostenible. Washington: BID, 2005. Disponible en Internet. http://www.iadb.org/ sds/doc/ENV-Checklist-ENV144E.pdf

BURNINGHAM, K.; FIELDING, J. \& THRUSH, D. 'It'll never happen to $\mathrm{me}^{\prime}$ : understanding public awareness of local flood risk. Disasters, 2008, vol. 32, № 2, p. 216-38.

CANALES, M. Métodos de investigación cualitativa. Santiago: Editorial Lom, 2006.

CISTERNAS, M.; ATWATER, B.; TORREJÓN, F.; SAWAI, Y.; MACHUCA, G.; LAGOS, M.; EIPERT, A.; YOULTON, C.; SALGADO, I.; KAMATAKI, T.; SHISHIKURA, M.; RAJENDRAN, C. P.; MALIK, J.; RIZAL, Y. \& HUSNI, M. Predecessors of the giant 1960 Chile earthquake. Nature, 2005, vol. 437, p. 404-407.

CHUQUISENGO, O. y GAMARRA, L. Propuesta metodológica para la gestión local de riesgos de desastre. Una experiencia práctica. Lima: ITDG, 2001. Disponible en Internet: http://bvpad.indeci.gob.pe/doc/pdf/ esp/doc414/doc414_contenido.pdf

CLARKE, C. y PINEDA. C. (eds.). Riesgo y desastres su gestión municipal en Centroamérica. Washington: BID, 2007. Disponible en Internet: http:// idbdocs.iadb.org/wsdocs/getdocument. aspx?docnum $=1320023$
COMISIÓN ECONÓMICA PARA AMÉRICA LATINA (CEPAL). Información para la gestión de riesgo de desastres. Estudio de caso de cinco países. Ciudad de México: ONU, BID, 2007. Disponible en Internet: http://www.crid.or.cr/digitalizacion/pdf/spa/ doc17340/doc17340.htm

CONVERTITO, V.; DE MATTEIS, R.; CANTORE, L.; A ZOLLO IANNACCONE, G. \& CACCAVALE, M. Rapid estimation of ground-shaking maps for seismic emergency management in the Campania Region of southern Italy. Natural Hazard, 2010, vol. 52, p. $97-115$

DIARIO LA NACIÓN. Falso tsunami: Penquistas volvieron a los cerros. Publicación periódica de Santiago, Chile, 18 de enero de 2005. Disponible en Internet: http:// www.Ianacion.cl/prontus_noticias/site/ artic/20050118/pags/20050118114311.html

DIARIO EL SUR. Falso tsunami: investigación en punto muerto: $A$ un año de pánico colectivo, aún no hay responsables. 18 de enero 2006. Disponible en internet: http://www.elsur.cl/edicion_hoy/secciones/ articulo.php? $\mathrm{id}=65839 \mathrm{ydia}=1137553200$

DIARIO EL DÍA. Tongoyinos vivieron horas de pánico con falso tsunami. Publicación periódica de La SerenaCoquimbo, Chile, 30 de junio de 2009. Disponible en Internet: http://www. diarioeldia.cl/index.php?option=com_content ytask=viewyid $=22834$ yltemid $=160$

DIARIO EL MOSTRADOR. Video de Onemi muestra descoordinación tras terremoto. Publicación periódica de Santiago, Chile, 14 de mayo de 2010. Disponible en Internet: http://www.elmostrador.cl/ multimedia/2010/05/14/video-de-onemimuestra-descoordinacion-tras-terremoto/

EDESO, J. Riesgos naturales geológicos y geomorfológicos. Lurralde: Investigacion y Espacio, 2008, vol. 31, p. 325-374.

ESTRATEGIA INTERNACIONAL PARA LA REDUCCIÓN DE DESASTRES (EIRD). MarCo de Acción de Hyogon para 2005-2015: aumento de la resiliencia de las naciones y 
las comunidades ante los desastres. Hyogo: EIRD, 2005. Disponible en internet: http:// www.unisdr.org/eng/hfa/docs/Hyogoframework-for-action-spanish.pdf

FERRADAS, P. Investigación, acción y participación en redes para la gestión de riesgos. En: INDECI. Nuevas perspectivas en la investigación científica y tecnológica para la prevención y atención de desastres: Memoria del Seminario Taller Internacional sobre Prevención y Atención de Desastres en la Educación. Lima: Instituto Nacional de Defensa Civil (INDECI), 2004, p. 24-26. Disponible en Internet: http://bvpad.indeci. gob.pe/doc/pdf/esp/doc849/doc849.htm

GARCÍA, J. y SILIÓ, F. Riesgos naturales en los Andes: cambio ambiental, percepción y sostenibilidad. Boletín de la A.G.E. 2001, No 30 , p. 69-84.

GÓMEZ, D. Ordenación del territorio. Una aproximación desde el medio físico. Madrid: Editorial Agrícola, 1994.

HALL, P. Early warning systems: reframing the discussion. The Australian Journal of Emergency Management, 2006, vol. 22, No 2 , p. 32-36.

I NSTITUTO NACIONAL DE ESTADÍSTICAS (INE). Censos de Población y Vivienda años 1960, 1970, 1982, 1992 y 2002 según país, Región de La Araucanía, provincias y comunas. Santiago: INE, 2003.

INTENDENCIA REGIÓN DE LA ARAUCANÍA (IRA). Plan regional de protección civil. República de Chile. Departamento de protección civil y social. Temuco: Gobierno interior Araucanía, 2004.

LAGOS, M. Tsunamis de origen cercano a las costas de Chile. Revista de Geografía Norte Grande, 2000, No 27, p. 93-102.

LAGOS, M.; CISTERNAS, M. y MARDONES, M. Construcción de viviendas sociales en áreas de riesgo de tsunami. Revista de la Construcción, 2008, vol. 7, № 2, p. 4-16.

LAGOS, M. y CISTERNAS, M. El nuevo riesgo de tsunami: considerando el peor escenario. Scripta Nova. Revista electrónica de geografía y ciencias sociales, 2008, vol. 12, No 270. Disponible en Internet: http:// www.ub.es/geocrit/-xcol/436.htm

LAGOS, M. y GUTIÉRREZ, D. Simulación del tsunami de 1960 en un estuario del centro sur de Chile. Revista de Geografía Norte Grande, 2005, №33, p. 5-18.

LAVELL, A. Desastres urbanos: una visión global. Lima: La Red, USAID, 2000. Disponible en Internet: http://www. desenredando.org/public/articulos/2000/ duurg/DUUVG_mar1-2002.pdf

MARDONES, M. y VIDAL, C. La zonificación y evaluación de los riesgos naturales de tipo geomorfológico: un instrumento para la planificación urbana en la ciudad de Concepción. EURE, Santiago, 2001, vol. 27, No 81, p. 97-122.

MITCHELL, A.; GLAVOVIC, B.; HUTCHINSON, B.; MACDONALD, G.; ROBERTS, M. \& GOODLAND, J. Community-based civil defence emergency management planning in Northland, New Zealand. Australasian Journal of Disaster and Trauma Studies, 2010, vol. 2010, N¹. Disponible en Internet: http://www.massey. ac.hz/ trauma/issues/2010-1/mitchell.htm

MONGE, J. Estudios de riesgo de tsunami en costas chilenas. Santiago, Chile. Jornadas chilenas de Sismología en ingeniería antisísmica, 1993, vol. 2, p. 3-22.

MONTENEGRO-ROMERO, T. Evaluación y zonificación del riesgo de inundación por tsunami como base para la planificación territorial del borde costero de la Novena Región de La Araucanía. Tesis de grado para optar al grado de Licenciado en Recursos Naturales. Temuco: Escuela de Ciencias Ambientales, Facultad de Recursos Naturales, Universidad Católica de Temuco, Chile, 2006.

MUNICIPALIDAD DE ANTOFAGASTA. Modificación del Plan regulador Comunal de Antofagasta Sector Norte. Antofagasta: Secretaría comunal de Planificación, 2008. Disponible en Internet: http://www. municipalidadantofagasta.cl/custom/ descargas/Ord.\%20Municipal\%20Plan\%20 Seccional\%20Norte.pdf 
MUNICIPALIDAD DE COQUIMBO. Plan Regulador intercomunal del borde costero de la región de Coquimbo. Etapa IV Ordenanza. Coquimbo: Ministerio de Viviendo y Urbanismo, 2005. Disponible en Internet: http://www.municipalidadantofagasta.cl/ index.php?option=com_contentyview $=$ article yid $=358$ yltemid $=153$

MUNICIPALIDAD DE VIÑA DEL MAR. Cuenta pública de la gestión de la alcaldesa y de la marcha general de la municipalidad año 2005. Viña del Mar: Municipalidadd de Viña del Mar, 2005. Disponible en Internet: http://www.munivina. $\mathrm{cl} /$ ?seccion $=5.5 y p g=0 y$ tipo $=\mathrm{CTA}$

NATHAN, F. Risk perception, risk management and vulnerability to landslides in the hill slopes in the city of La Paz, Bolivia. A preliminary statement. Disasters, 2008, vol. 32, $\mathrm{N}^{\circ} 21$, p. 337-357.

OFICINA NACIONAL DE EMERGENCIA (ONEMI). Simulacro de tsunami en Puerto Saavedra (octubre). Santiago: ONEMI, inédito, 1999.

OFICINA NACIONAL DE EMERGENCIA (ONEMI). Metodología para la gestión integral y participativa de prevención, preparación, respuesta y recuperación frente a emergencias y desastres, de acuerdo a las específicas realidades locales de riesgos y de recursos (AIDEP). Santiago: Departamento de Protección civil, ONEMI, 2001.

OFICINA NACIONAL DE EMERGENCIAS (ONEMI). Plan nacional de Emergencia. Santiago: Ministerio del Interior, República de Chile, 2002. Disponible en Internet: http://www.onemi.cl/images/onemi/archivos/ decretoplannacionalprotcivil.pdf.

ORGANIZACIÓN PANAMERICANA DE LA SALUD/ORGANIZACIÓN MUNDIAL PARA LA SALUD (OPS/OMS). Condiciones de riesgo en América Latina y el Caribe. Washington: OPS/OMS, 1994. Disponible en Internet: http://www.helid.desastres. net/?e=d-010who--000--1-0--010---4-----0-0-10l--11 en-5000---50-about-0---01131-001110utfZz-8-0-0ya $=$ dyc $=$ whoyc $\mathrm{l}=\mathrm{CL} 1.3 \mathrm{yd}=\mathrm{J} 0$ 52.5.pr
PEÑA-CORTÉS, F.; HAUENSTEIN, E., DURÁN, T.; BERTRÁN, C.; SCHLATTER, R. y TAPIA J. Informe de avance Proyecto FONDECYT No 1030861. Análisis Integrado del Borde Costero de la IX Región. Propuestas y Criterios para la Planificación Ecológica de sus Humedales. Temuco: inédito, 2004.

PEÑA-CORTÉS, F.; REB OLLEDO, G.; HERMOSILLA, K.; HAUENSTEIN, E; BERTRÁN, C.; SCHLATTER, R. y TAPIA, J. Dinámica del paisaje para el periodo 19802004 en la cuenca costera del río Budi, Chile. Consideraciones para la conservación de sus humedales. Ecología Austral, 2006, № 16, p. 183-196.

PEÑA-CORTÉS, F.; AILIO, C.; GUTIÉRREZ, P.; ESCALONA-ULLOA, M.; REBOLLEDO, G.; PINCHEIRA-ULBRICH, J.; ROZAS, D. y HAUENSTEIN, E. Morfología y dinámica dunaria en el borde costero de la región de La Araucanía en Chile. Antecedentes para la conservación y gestión territorial. Revista de Geografía Norte Grande, 2008, № 41, p. 63-80.

PEÑA-CORTÉS, F.; ESCALONA-ULLOA, M.; REBOLLEDO, G.; PINCHEIRA-ULBRICH, J.y TORRES-ÁLVAREZ, O. Efecto del cambio en el uso del suelo en la economía local: una perspectiva histórica en el borde costero de La Araucanía, Sur de Chile. En: CONFALONIERI, U.; MENDOZA, M. y FERNÁNDEZ, L. (eds.). Efecto de los cambios globales sobre la salud humana y la seguridad alimentaria. Buenos Aires: RED CYTED 406RT0285, 2009, p. 184-197.

RAMÍREZ, J. Tsunami. Revista Geográfica de Chile. Terra Australis, 1986, № 29, p. 29-39.

SANTIAGO, C. y SAGÁSTEGUI, W. Preparados contra los desastres. Una experiencia de prevención y respuesta a emergencias en comunidades nativas y mestizas en la región amázonica del Perú. Lima: Soluciones Prácticas-ITDG, 2008.

SERVICIO HIDROGRÁFICO Y OCEANOGRÁFICO DE LA ARMADA DE CHILE (SHOA). Cómo Sobrevivir a un Maremoto". Santiago: SHOA, 2000a. 
SERVICIO HIDROGRÁFICO Y OCEANOGRÁFICO DE LA ARMADA DE CHILE (SHOA). El Maremoto del 22 de mayo de 1960 en las costas de Chile. Valparaíso: SHOA, 2000b.

SERVICIO HIDROGRÁFICO Y OCEANOGRÁFICO DE LA ARMADA DE CHILE (SHOA). Instrucciones oceanográficas $N^{o}$ 3. Instrucciones generales sobre el sistema nacional de alarma de maremotos. Valparaíso: SHOA, 2001.

SIEGRIST, M. \& HEINS, G. Flooding risks: a comparison of lay people's perceptions and expert's assessments in Switzerland. Risk Analysis, 2006, vol. 26, № 4, p. 971-979.

SIEVERS, H.; VILLEGAS, G. \& BARROS, G. The seismic sea wave of 22 may 1960 along the Chilean Coast. Seismological Society of America, Bulletin, 1963, vol. 53, p. 1125-1190.
VEYL, C. Los sismos y las erupciones de mayo de 1960 en el sur de Chile. Sociedad Chilena de Química, Boletín, 1961, vol. 11, $N^{\circ} 1-2$, p. 20-32.

VIDAL, C.; LAGOS, L.; ESPINOSA, J.; ARANCIBIA, V. y YÁÑEZ, M. Aportes al ordenamiento territorial urbano: Evaluación de riesgos naturales en Concepción y aplicación en SIG. Urbano, 2001, vol. 4, No 5, p. 56-62.

WAMSLER, C. Integrando la gestión del riesgo, planificación urbana y vivienda social: lecciones de El Salvador. Revista INVI, 2007, No 59, p. 93-114.

ZHAI, G. \& IKEDA, S. Flood risk acceptability and economic value of evacuation. Risk Analysis, 2006, vol. 26, Nº 3, p. 683-694.

ZOU, L. \& WEI Y. Impact assessment using DEA of coastal hazardson socialeconomy in Southeast Asia. Natural Hazard, 2009, vol. 48, p. 167-189. 